\title{
Sustained bile drainage decreases the organs injuries via inflammation-associated factors modulation in a severe acute pancreatitis rat model
}

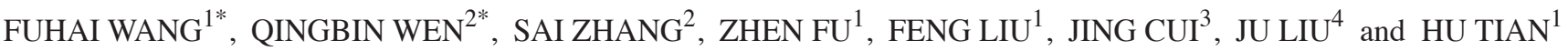 \\ ${ }^{1}$ Department of General Surgery, Shandong Provincial Qianfoshan Hospital, Shandong University; \\ ${ }^{2}$ Department of Surgery, Shandong University of Traditional Chinese Medicine; ${ }^{3}$ Department of Pathology, \\ Shandong Provincial Qianfoshan Hospital, Shandong University; ${ }^{4}$ Laboratory of Microvascular Medicine, \\ Medical Research Center, Shandong Provincial Qianfoshan Hospital, Shandong University, \\ Jinan, Shandong 250014, P.R. China
}

Received July 11, 2018; Accepted March 7, 2019

DOI: $10.3892 /$ etm.2019.7478

\begin{abstract}
The timely and effective treatment for severe acute pancreatitis (SAP) is favorable to prognosis. Decompression of the bile duct might be a feasible way to decrease the progression of SAP. The present study investigated the effects of sustained bile external drainage on organs injury caused by SAP in Sprague-Dawley (SD) rats and the mechanisms involved. A total of 72 female SD rats weighting 190-230 g were randomly divided into four groups $(n=18)$ : Sham operation group (SOG), SOG + bile drainage group (BDG), SAP group, and $\mathrm{SAP}+\mathrm{BDG}$. Sodium taurocholate solution $(4 \% ; 1 \mathrm{mg} / \mathrm{kg}$ body weight) was used to set up SAP model via injection of retrograde puncture of biliopancreatic duct through the duodenum. A cannula was inserted into the bile duct and fixed externally to establish BDG model. At each time points $(t=3,6,12 ; n=6)$, tissues from the liver, lung, and pancreas, and blood samples were collected. Serum amylase (AMY) was analyzed in all the samples. The levels of tumor necrosis factor- $\alpha$ (TNF- $\alpha$ ), heme oxygenase-1 (HO-1), interleukin-10 (IL-10) and high mobility group box 1 (HMGB1) were detected by ELISA. Hematoxylin-eosin staining was performed to observe the histopathological changes, and nuclear transcription factor (NF)- $\mathrm{B}$-p65 levels in the pancreas were analyzed by western blotting. The data indicated that BDG alleviated the SAP progression and multiple organs injuries. Meanwhile, the histopathological changes of the pancreas, liver, and lungs were
\end{abstract}

Correspondence to: Dr Hu Tian, Department of General Surgery, Shandong Provincial Qianfoshan Hospital, Shandong University, 16766 Jingshi Road, Jinan, Shandong 250014, P.R. China

E-mail: tianhu6585@163.com

*Contributed equally

Key words: bile drainage, severe acute pancreatitis, inflammationassociated factors, organs injury, nuclear transcription factor- $\kappa \mathrm{B}-\mathrm{p} 65$ improved by BDG. BDG decreased the pathological scores of pancreas significantly $(\mathrm{P}<0.05)$. The levels of AMY, TNF- $\alpha$, HMGB1, and NF-кB-p65 were significantly downregulated by BDG $(\mathrm{P}<0.05)$, while the level of $\mathrm{HO}-1$ was upregulated and IL-10 was unchanged. In summary, BDG may attenuate the multiple organs injuries caused by SAP via downregulation of TNF- $\alpha$, HMGB1, NF- $\kappa$ B-p65 and upregulation of HO-1.

\section{Introduction}

Severe acute pancreatitis (SAP) is characterized by rapid progression, severe systemic complications, multiple organs dysfunction, and high mortality (1). The activation of pancreatic enzymes, release of inflammatory factors, and subsequent systemic inflammatory response syndrome (SIRS) and multiple organ dysfunction syndromes (MODS) are the main pathological processes of SAP. The bile reflux deprived from biliary obstruction or the pancreatic duct obstruction (or increased pressure in the pancreatic duct) is considered as the most important factor for developing SAP (2). Targeted treatment such as sphincterotomy or nasobiliary drainage may prevent the progression of acute pancreatitis (AP) or SAP $(3,4)$, which indicates that sustained bile drainage or biliary decompression could decrease the organ injury in SAP and improve the prognosis.

Exploration for the mechanisms of SAP progression and the relevant treatment strategies are particularly important. Inflammation-associated factors serve a vital role in the progression of SAP. The imbalance between pro-inflammatory factors including tumor necrosis factor (TNF)- $\alpha$ and high mobility group box 1 (HMGB1) and anti-inflammatory factors like hemeoxygenase-1 (HO-1) is indispensable for SAP progression, which leads to local pancreatic inflammation and systemic complications (5-7). Previous studies have demonstrated that TNF- $\alpha$ and interleukin-6 (IL-6) activate the Kupffer cells in the liver and then produce pro-inflammatory factors, thus inducing early liver injury, and gut-derived cytokines reaching the lung, resulting in acute respiratory distress syndrome (8-11). TNF- $\alpha$ affects the kidney function directly 
or indirectly via cytotoxicity or by inducing the release of endothelin, respectively (12).

A previous study has demonstrated that biliary tract external drainage protected multiple organs against SAP associated injuries via HO-1 upregulation (7). HO-1 is also demonstrated to modulate some immunoinflammatory and autoimmune diseases, such as multiple sclerosis, which may represent a novel treatment approach for the above diseases $(13,14)$. Furthermore, the antioxidant factor nuclear factor E2-related factor 2 could also promote anticancer activity via the modulation of HO-1 (15). Nuclear transcription factor (NF) $-\kappa \mathrm{B}$ is an important pro-inflammatory factor and $\mathrm{NF}-\kappa \mathrm{B}-\mathrm{p} 65$ or RelA is a member of NF- $\kappa \mathrm{B}$ family, which is associated with cell proliferation and apoptosis $(16,17)$. Based on the previous studies, the present study aimed to further investigate the role of sustained bile external drainage in decreasing the injury of SAP and the association of sustained bile drainage group (BDG) on inflammatory factors TNF- $\alpha$, HMGB1, IL-10, NF- $\kappa$ B-p65, and HO-1 in SAP rats at different periods $(3,6$ and $12 \mathrm{~h})$. The present study provided evidence for the application of sustained BDG in the treatment of SAP.

\section{Materials and methods}

Reagents. Sodium taurocholate was from Sigma-Aldrich; Merck KGaA (purity >97\%). TNF- $\alpha$ (JYM0635Ra), HO-1 (JYM0356Ra), IL-10 (JYM0651Ra), and HMGB1 (JYM0371Ra) ELISA kit were all purchased from ColorfulGene Biological Technology, NF-кB-p65 mouse antibody (8242S) was from Cell Signaling Technology, Inc.

Establishment of animal model. A total of 72 8-week-old female Sprague-Dawley (SD) rats weighing 190-230 g were randomly divided into four groups $(n=18)$ : Sham operation group (SOG), SOG + bile drainage group (BDG), SAP group, and SAP + BDG. The animals (6 weeks of age) were purchased from Jinan Peng Yue Laboratory Animal Breeding Company and fed with food and water freely under the same environment at $22-26^{\circ} \mathrm{C}$ for 2 weeks with a $12 \mathrm{~h}$ light/dark cycle. The present study was approved by the Ethics Committee of Shandong Provincial Qianfoshan Hospital affiliated to Shandong University.

The SD rats received intraperitoneal anesthesia with $3 \%$ pentobarbital sodium (30 mg/kg body weight). The duodenum, bile duct, and pancreas were separated, and $4 \%$ sodium taurocholate solution was injected by retrograde puncture of biliopancreatic duct through duodenum to set up the SAP model ( $1 \mathrm{mg} / \mathrm{kg}$ body weight). The SOG group received operation only without any other interventions. After the injection of sodium taurocholate, a cannula of diameter $0.8 \mathrm{~mm}$ was inserted into the bile duct through duodenum. The cannula was then fixed with the bile duct, duodenum and abdominal wall separately when the drainage of the bile was unobstructed to establish SAP + BDG model. The SOG + BDG group only fixed a cannula into bile duct without the application of $4 \%$ sodium taurocholate solution. Pentobarbital sodium ( $200 \mathrm{mg} / \mathrm{kg}$ body weight) with an intraperitoneal injection was used to sacrifice the rats prior to blood collection from the abdominal aorta and the tissue samples were collected.
Analysis of serum amylase (AMY). The blood samples (5 ml) were obtained at various time points $(\mathrm{t}=3 \mathrm{~h}, 6 \mathrm{~h}, 12 \mathrm{~h})$ from abdominal aorta. After standing for $15 \mathrm{~min}$ at room temperature, the samples were centrifuged at $1,370 \mathrm{x} g$ for $10 \mathrm{~min}$ at $4^{\circ} \mathrm{C}$. The serum supernatant was collected. The level of serum AMY was detected in the clinical laboratory of Qianfoshan Hospital (7).

$H \& E$ staining. Tissue samples of pancreas, liver, and lung were collected at various time points ( $\mathrm{t}=3 \mathrm{~h}, 6$, and $12 \mathrm{~h})$ and immersed in $10 \%$ paraformaldehyde and paraffin embedded. Thick tissue sections (4- $\mu \mathrm{m})$ were obtained. Subsequently, the sections were dewaxed in xylene and dehydrated through a serial alcohol gradient followed by washing with PBS for $5 \mathrm{~min}$ at room temperature. The sections were stained with hematoxylin for $5 \mathrm{~min}$ and eosin for $30 \mathrm{sec}$ at room temperature. Following the staining, sections were dehydrated by increasing concentrations of ethanol and xylene. The histomorphology was observed with an optical microscope (magnification, $\mathrm{x} 200$ ). The evaluation standard of histopathologic scores of the pancreas were recorded in Table I (18). The current study selected three sections and five fields (magnification, x200) for every section randomly. The scores were subsequently calculated in accordance with the standard of table I for every field in four parts (edema, inflammation, hemorrhage and necrosis). The average value for the histopathologic scores of the pancreas were then calculated.

ELISA. The SD rats were divided into four groups: SOG, $\mathrm{SOG}+\mathrm{BDG}, \mathrm{SAP}$ group, and SAP + BDG. Blood $(5 \mathrm{ml})$ from the abdominal aorta was collected at various time points $(\mathrm{t}=3 \mathrm{~h}, 6$, and $12 \mathrm{~h}$ ) in every group. After $15 \mathrm{~min}$, the blood was centrifuged at $1,370 \mathrm{x}$ g for $10 \mathrm{~min}$ at room temperature and serum supernatants were collected. Measurement of TNF- $\alpha$, HO-1, IL-10, and HMGB1 levels in blood supernatants was performed using commercially available ELISA kits (ColorfulGene) according to the manufacturer's protocol. Optical density (OD) values were obtained at $450 \mathrm{~nm}$ using the Spectra Max 190 (Molecular Devices, LLC).

Western blotting. The SD rats were divided into four groups as stated above; the pancreas tissues were collected and lysed in cold radio immunoprecipitation assay lysis buffer (Beyotime Institute of Biotechnology) with $1 \mathrm{nM}$ phenylmethylsufonyl fluoride for $30 \mathrm{~min}$ on ice, followed by centrifugation at $16,000 \mathrm{x} \mathrm{g}$ for $15 \mathrm{~min}$ at $4^{\circ} \mathrm{C}$. Protein concentrations of cleared lysates were determined with a bicinchoninic acid Protein Assay kit (Beyotime Institute of Biotechnology). Total amount of proteins $(40 \mu \mathrm{g})$ were separated on $10 \% \mathrm{SDS}$-polyacrylamide gel electrophoresis gels (Beyotime Institute of Biotechnology) and then electrotransferred to a polyvinylidene fluoride (PVDF) membrane. PVDF membranes were blocked with $5 \%$ skimmed milk and incubated overnight at $4{ }^{\circ} \mathrm{C}$ with the NF- $\kappa$ B-p65 primary antibody (cat. no. 8242S; 1:1,000; Cell Signaling Technology, Inc.) and GADPH primary antibody (cat. no. ab37168; 1:1,000; Abcam) in PBS-Tween. Following three washes with PBS containing 0.1\% Tween-20 for 15 min each, the membranes were incubated with horse radish peroxidase-conjugated anti-rabbit IgG (cat. no. A0280; 1:1,000; Beyotime Institute of Biotechnology) at $37^{\circ} \mathrm{C}$ for $1 \mathrm{~h}$ and then 
Table I. Histologic scoring for acute hemorrhagic necrotizing pancreatitis.

\begin{tabular}{lcl}
\hline Condition & Score & \multicolumn{1}{c}{ Description } \\
\hline Edema & 0 & Absent \\
& 1 & Focally increased between lobules \\
& 2 & Diffusely increased between lobules \\
& 3 & Tense acini, widely separated lobules \\
& 4 & Gross lobular separation \\
Inflammation & 0 & Absent \\
& 1 & Around ductal margins \\
& 2 & In parenchyma (<50\% of lobules) \\
& 3 & In parenchyma (51 to 75\% of lobules) \\
Hemorrhage & 4 & Massive collections, abscesses \\
& 0 & Absent \\
& 1 & Blood in parenchyma $(<25 \%)$ \\
& 2 & Blood in parenchyma $(25$ to 50\%) \\
& 3 & Blood in parenchyma $(50$ to $75 \%)$ \\
Necrosis & 4 & Blood in 100\% of lobules \\
& 0 & Absent \\
& 1 & Periductal parenchymal destruction \\
& 2 & Focal parenchymal necrosis $(<20 \%)$ \\
& 3 & Diffuse loss of lobules (20 to 50\%) \\
& 4 & Severe loss of lobules $(>50 \%)$
\end{tabular}

washed three times with PBS containing 0.1\% Tween-20. A TANON-4500SF chemiluminescence system (Tanon Science and Technology Co., Ltd, Shanghai, China) was used to detect the target proteins.

Statistics. All the data are expressed as the mean \pm standard deviation. Multiple group comparisons of the means were carried out by one-way analysis of variance followed by the least significant difference test as the post hoc test. Statistical analyses were performed with SPSS software (version 17.0, SPSS China). $\mathrm{P}<0.05$ was considered to indicate a statistically significant difference.

\section{Results}

Effects of sustained BDG on serum levels of TNF- $\alpha, H O-1$, $I L-10$, and $H M G B 1$. To explore the effect of BDG on pancreas in SAP, the SAP rat models were induced by $4 \%$ sodium taurocholate solution via injection of retrograde puncture of biliopancreatic duct through duodenum, and the pancreas and intestine were obviously edematous while ascites existed in the SAP rats (Fig. 1A, right panel). The serum levels of AMY in the SAP group and SAP + BDG group were higher compared with the SOG group and SOG + BDG group, while BDG could decrease the level of AMY in SAP (Fig. 1B). SIRS is the main reason for high mortality at the early stage of SAP, the inflammatory cytokines induce the cascade effect, even the MODS and death (19). The pro-inflammatory factors TNF- $\alpha$ and HMGB1 were upregulated in SAP rats, however sustained BDG could decrease the level of TNF- $\alpha$ in SAP significantly at different times, while HMGB1 was only downregulated
A

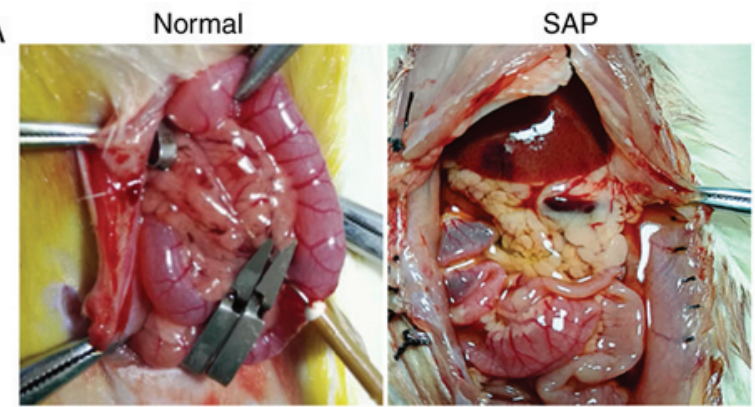

B

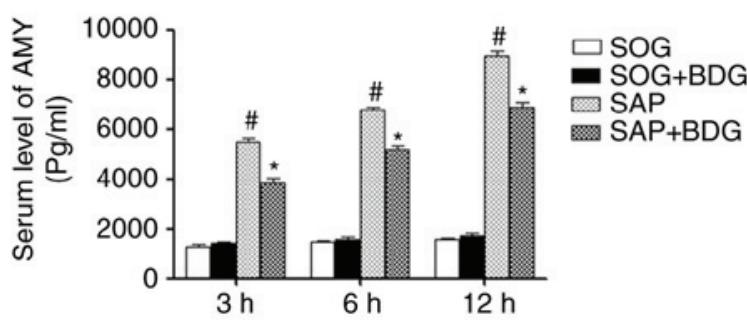

Figure 1. Establishment of SAP rat model and analysis of serum AMY. (A) sodium taurocholate solution $(4 \% ; 1 \mathrm{mg} / \mathrm{kg}$ body weight) was used to set up the SAP model via injection of retrograde puncture of biliopancreatic duct through duodenum. (B) The serum levels of AMY were detected at 3,6 , and $12 \mathrm{~h}(\mathrm{n}=6) .{ }^{\#} \mathrm{P}<0.05$ vs. SAP + BDG; ${ }^{*} \mathrm{P}<0.05$ vs. SOG + BDG. AMY, amylase; BDG, bile drainage group; SAP, severe acute pancreatitis; SOG, sham operation group.

at the $6 \mathrm{~h}$ of SAP by BDG, and no significant differences were observed at 3 and $12 \mathrm{~h}$ (Fig. $2 \mathrm{~A}$ and C). There was no marked difference in the serum level of IL-10 in the SAP and SAP + BDG groups (Fig. 2D). The anti-inflammatory factor HO-1 was increased in SAP groups at early stages ( 3 and $6 \mathrm{~h}$ ), but decreased at $12 \mathrm{~h}$, and then sustained BDG upregulated the serum level of HO-1 compared with the SAP group at different time check-points of SAP (Fig. 2B).

The effect of sustained BDG on the multiple organ injury of SAP. To evaluate the effect of BDG, the histopathologic changes of pancreas, liver, and lung were observed. No significant histopathologic changes in the SOG and SAP+SOG groups were observed (Fig. S1). The inflammatory cells infiltration, cell necrosis, the damage of pancreatic lobule was higher in the SAP group, and obvious hemorrhage and tissue necrosis in a time-dependent manner was observed, while BDG significantly mitigated the abovementioned phenomena (Fig. 3A). The histopathologic scores of pancreas in SAP were higher than the SOG groups, while BDG decreased the histopathologic scores of SAP significantly (Fig. 3B). Furthermore, compared with the SAP + BDG group the liver cells edema, necrosis, hepatic cord disorder, and inflammatory cells infiltration were more obvious in SAP at all time points (Fig. 4A). Finally, the edema and hyperemia of lung tissue, the red blood cells and inflammatory cells infiltration in alveolar cavity and interstitial tissue were more serious than the SAP + BDG group (Fig. 4B).

The modulation by $B D G$ on the expression level of $N F-\kappa B-p 65$ in the pancreas. During subsequent experiments, the rat pancreas tissues at different time points was collected. The

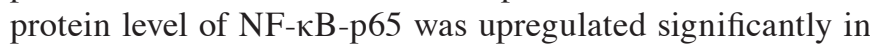



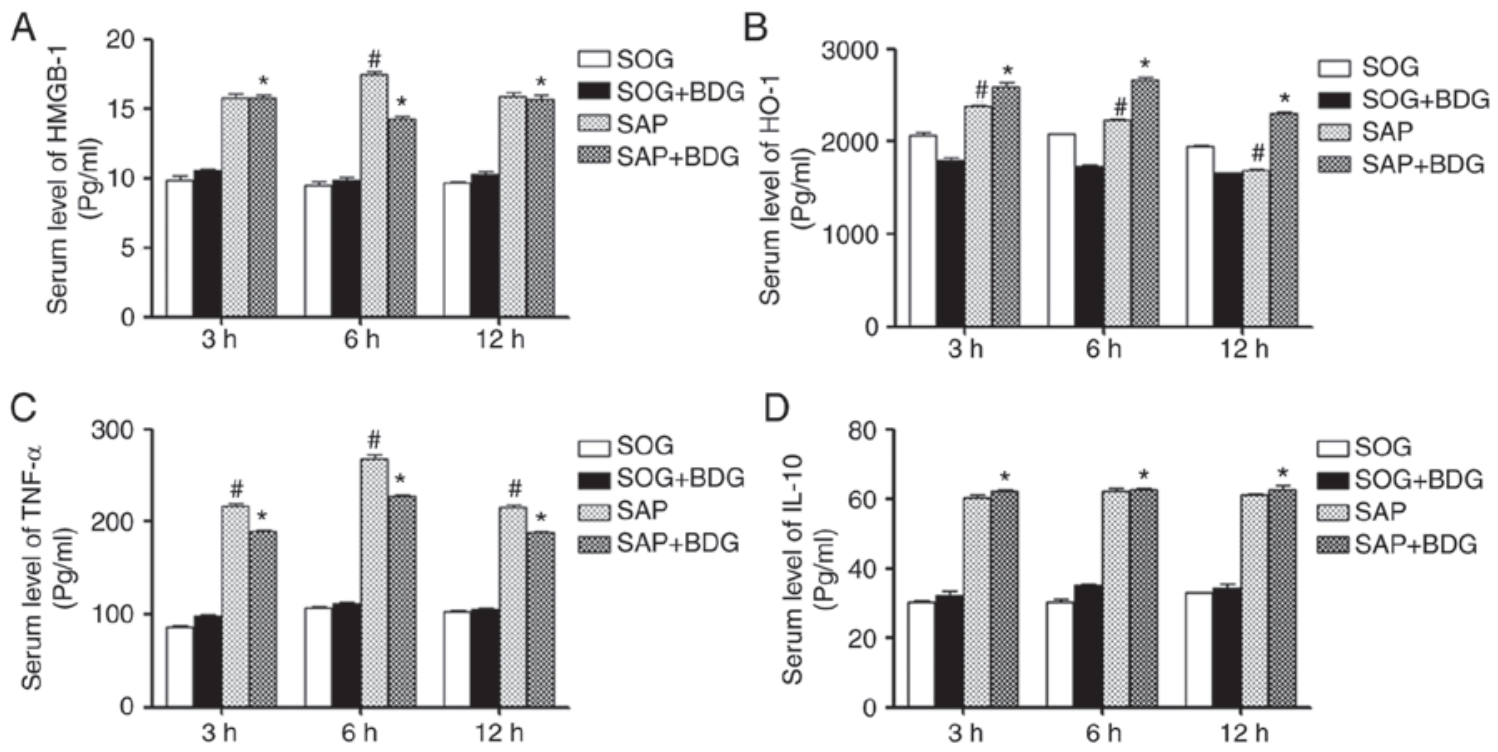

Figure 2. Effect of sustained bile drainage on serum expression of inflammatory factors. The Sprague-Dawley rats were divided into four groups: SOG, SOG + BDG, SAP, and SAP + BDG. The serum levels of (A) HMGB1, (B) HO-1, (C) TNF- $\alpha$ and (D) IL-10 were detected by ELISA assay at 3, 6, and $12 \mathrm{~h}$ (n=6). ${ }^{\#} \mathrm{P}<0.05$ vs. SAP + BDG; ${ }^{\text {P }}<0.05$ vs. SOG + BDG. BDG, bile drainage group; HMGB1, high mobility group box 1; HO-1, heme oxygenase-1; IL-10, interleukin-10; SAP, severe acute pancreatitis; SOG, sham operation group; TNF- $\alpha$, tumor necrosis factor- $\alpha$.
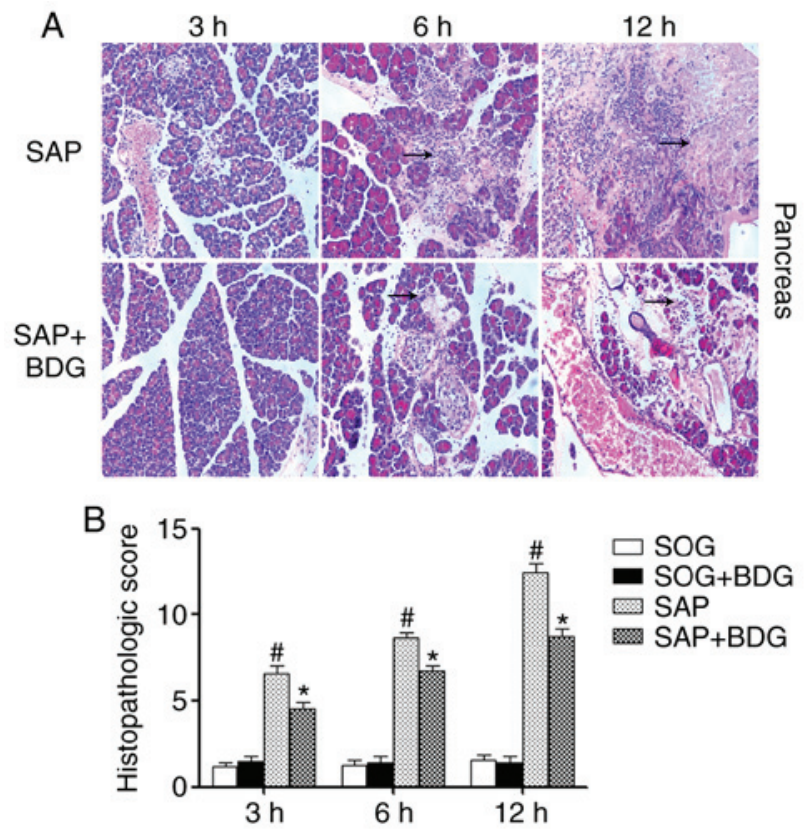

Figure 3. Histopathologic changes and score of the pancreas. The pancreatic tissues of the SAP and SAP + BDG groups were collected at 3,6, and $12 \mathrm{~h}$ (A) H\&E staining (black arrow) and (B) histopathologic scores were operated. ${ }^{\# P}<0.05$ vs. SAP + BDG; ${ }^{*} \mathrm{P}<0.05$ vs. SOG + BDG. BDG, bile drainage group; SAP, severe acute pancreatitis; SOG, sham operation group.

the SAP group in a time-dependent manner, while no marked difference was found in the SOG group (Fig. 5A). To further explore the role of BDG, the expression level of NF- $\mathrm{kB}-\mathrm{p} 65$ at every time point was monitored. The results indicated that the NF- $\mathrm{KB}-\mathrm{p} 65$ expression levels of the SOG and SOG + BDG groups were similar and those of SAP were the highest, among all groups tested. BDG downregulated its expression compared with the SAP group significantly at different time points (Fig. 5B).
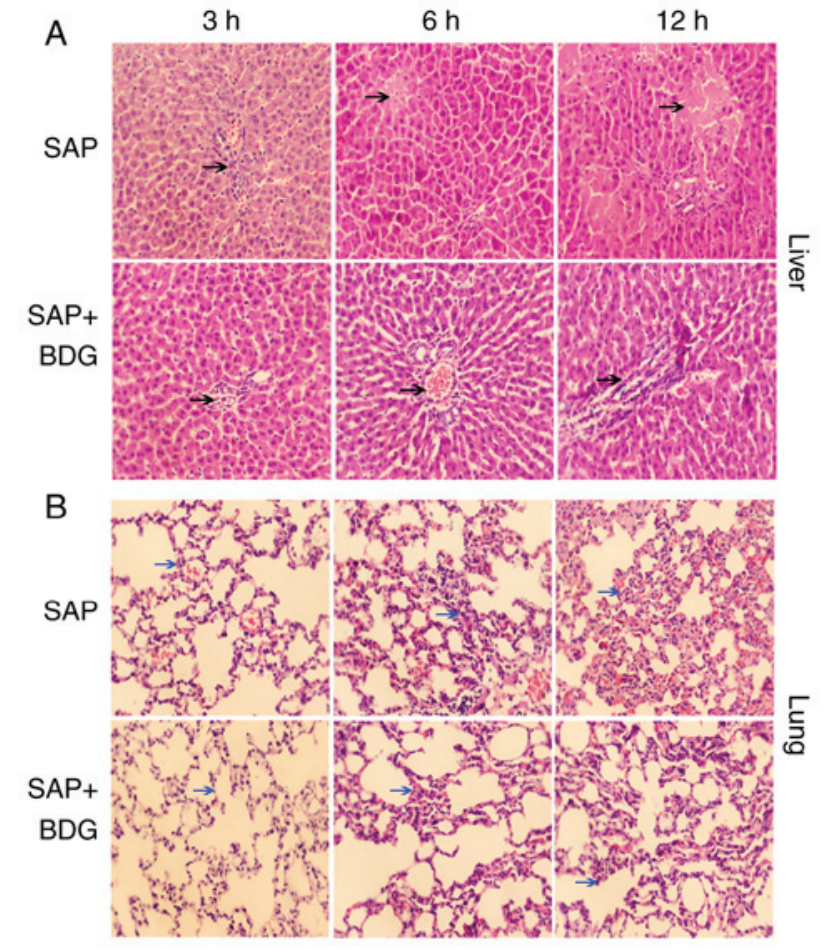

Figure 4. Histopathologic changes of the liver and the lung. The (A) liver and (B) lung tissues were collected at 3, 6, and $12 \mathrm{~h}$ in the SAP and SAP + BDG groups. H\&E staining was used to observe the histopathologic changes (black arrow, liver; blue arrow, lung; magnification, x200). BDG, bile drainage group; SOG, sham operation group.

\section{Discussion}

Severe acute pancreatitis (SAP) is a type of severe digestive disease associated with rapid progression, severe complications, and high morality. Blockage of the biliopancreatic duct, alcohol consumption, infection, and medical injury are the 


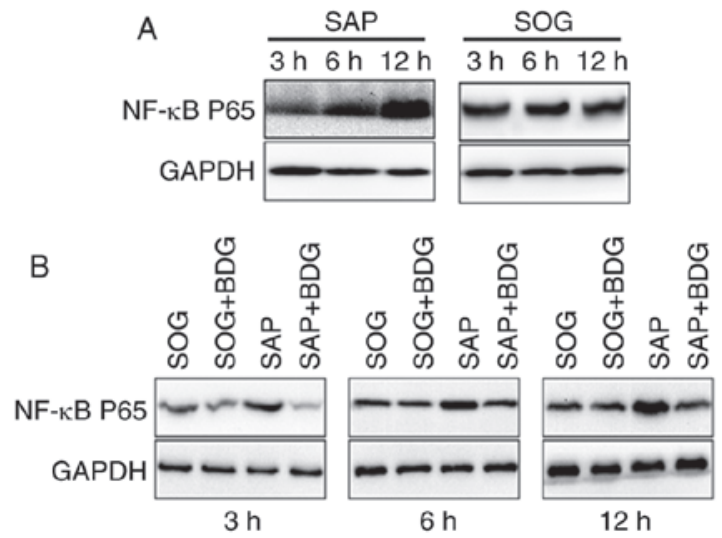

Figure 5. Early bile external drainage decreases the expression of NF-кB-p65 in the pancreas. (A) The expression levels of NF- $\kappa \mathrm{B}-\mathrm{p} 65$ was detected in the SAP and SOG groups at 3,6, and $12 \mathrm{~h}$. (B) Western blotting was used to analyze the NF-kB-p65 expression levels in the SOG, SOG + BDG, SAP, and $\mathrm{SAP}+\mathrm{BDG}$ groups at various time points. $\mathrm{BDG}$, bile drainage group;

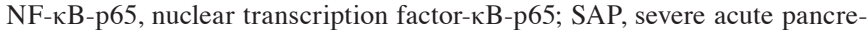
atitis; SOG, sham operation group.

common reasons for SAP (20). The mechanisms of SAP are complex. The activation of pancreatic enzymes, the release of inflammatory factors, oxidative stress reaction, translocation of gut microflora, and effect of nitric oxide are responsible for the progress of SAP (21-23). The imbalance between pro-inflammatory factors (TNF- $\alpha$, IL-6, and HMGB1) and anti-inflammatory factors (IL-2 and IL-10) results in the cascade effect, and finally leads to multiple organ injury and poor prognosis $(24,25)$.

Bile reflux activates pancreatic enzymes, and the degree of bile duct blockage is positively associated with the degree of AP. Previous studies have demonstrated that removal of bile or pancreatic duct obstruction effectively alleviated the progress of SAP $(4,7,26)$. In animal experiments, bile drainage prolonged the survival and relieved the pathological damage to the intestine (27). Hypoperfusion induced the liver to release inflammatory factors, TNF- $\alpha$, HMGB1, and IL-6 into the bile, and further aggravates the injury of intestinal mucosa (28). Previous studies demonstrated that bile external drainage protected the intestine and organ functions under shock via blocking the 'gut-liver-lung' cytokines axis, and decreased the injury to liver and lungs $(7,29)$, which is consistent with the present study. Meanwhile, biliary drainage is not indicated for chronic non-progressive elevation of SAP, but for a progressive increase in SAP or persistent jaundice (30). In the present study, based on the H\&E staining, it was found that the inflammation, cell necrosis and organs structure damage were aggravated with the progress of SAP in a time-dependent manner, and sustained BDG improved above phenomena significantly and exhibited multiple organs protective effects. The present study indicated that bile drainage or biliary decompression could alleviate the progress of SAP and protect the organs, and decrease the possibility of SIRS and MODS, which could be considered as a potential treatment choice for SAP. Future studies exploring the underlying mechanisms or signaling pathways are required to provide more evidence for the clinical application of sustained BDG.
A previous study reported that ascites induced the secretion of TNF- $\alpha$ in pancreatic acinar cells (31). TNF- $\alpha$ mRNA was overexpressed in pancreas tissues of SAP rat models, and the phenomenon was also observed in the liver and lungs along with the progress of SAP (32). Another pro-inflammatory factor, HMGB1, is also crucial for the pathogenesis of SAP. HMGB1 could activate toll-like receptors 4 (TLR4) signaling pathway, which is indispensable for the inflammatory process, and the specific inhibitors of HMGB1 or TLR4 (such as eritoran and [(S,R)-3-phenyl-4,5-dihydro-5-isoxasole acetic acid)] could alleviate the inflammation and improve the prognosis (33-35). The present study revealed that TNF- $\alpha$ and HMGB1 were upregulated in the SAP and SAP + BDG groups compared with the control groups, but BDG decreased the levels of TNF- $\alpha$ significantly and HMGB1 at $6 \mathrm{~h}$. HO- 1 is considered as an important anti-inflammatory factor, which is responsible for cytothesis and tissue protection (36). HO-1 could exert anti-inflammatory effects via the production of carbon monoxide (CO), which may be a possible factor of early treatment of SAP, while $\mathrm{CO}$ has been proven effective in some immuno-inflammatory and autoimmune diseases $(37,38)$. Under stress, HO-1 significantly inhibits apoptosis of the islet cells and protects the pancreatic $\beta$-cells $(39,40)$. It was demonstrated that HO-1 levels were increased in both SAP groups, and BDG upregulated the serum level of HO-1 compared with the SAP group. The present study demonstrated that sustained BDG could decrease the inflammation process by modulating the expressions of TNF- $\alpha, \mathrm{HO}-1$ and HMGB1, and alleviate the degree of SIRS and SAP model organ injury. However, SOG+BDG group could not return SAP levels to normal compared with SOG group, while BDG is considered as an effective method to slow the progress of SAP. Furthermore, other clinical strategies, including the inhibition of pancreatin, nutritional support and fasting, are also indispensible $(1,4)$. Meanwhile, the associated signaling pathways involved in the above inflammatory factors still remain to be explored. NF- $\mathrm{\kappa B}$ is crucial for the inflammatory activities via pro-inflammatory cytokines release. Picroside II is demonstrated to reduce the level of NF- $\mathrm{kB}$ and autophagy, and improves the antioxidant and anti-inflammatory activities of SAP models (41). The present study demonstrated that the NF- $\kappa$ B-p65 level of SAP was upregulated, and BDG downregulated the expression of NF- $\mathrm{kB}-\mathrm{p} 65$ significantly. According to the modulation of NF- $\mathrm{KB}-\mathrm{p} 65$, bile drainage could decrease the release of pro-inflammatory cytokines. However, whether TNF- $\alpha$ and HMGB1 are the downstream factors still remains unclear. The activation of NF- $\mathrm{KB}$ signaling pathway is important for the progress of SAP, and it is indicated that sustained BDG could decrease the activity of NF- $\mathrm{KB}$ signaling and further improve the prognosis of SAP.

In conclusion, the present study demonstrated that BDG decreased the progression of SAP effectively. The histopathologic changes of pancreas, liver, and lung were significantly attenuated by BDG. It was highlighted that the underlying mechanisms were the downregulation of TNF- $\alpha, N F-\kappa B$ p65 and HMGB1 and upregulation of HO-1 modulated by BDG.

\section{Acknowledgements}

Not applicable. 


\section{Funding}

The present study was supported by grants from Science and Technology Development Plan of Jinan City (grant no. 20140821), Key Research and Development Plan of Shandong Province (grant no. 2016GSF201108), and Science and Technology Development Plan of Shandong Province (grant no. 2011YD18017).

\section{Availability of data and materials}

The analyzed data sets generated during the study are available from the corresponding author on reasonable request.

\section{Authors' contributions}

HT and JL conceived, designed and supervised the present study. JC and ZF developed the methodology. FW, QW, SZ and FL performed the study and acquired the data. FW and QW reviewed the manuscript and analyzed/interpreted the data.

\section{Ethics approval}

The present study was approved by the Ethics Committee of Shandong Provincial Qianfoshan Hospital affiliated to Shandong University.

\section{Patient consent for publication}

Not applicable.

\section{Competing interests}

The authors declare that they have no competing interests.

\section{References}

1. Bai Y, Liu Y, Jia L, Jiang H, Ji M, Lv N, Huang K, Zou X, Li Y, Tang C, et al: Severe acute pancreatitis in China: Etiology and mortality in 1976 patients. Pancreas 35: 232-237, 2007.

2. Alexakis N, Lombard M, Raraty M, Ghaneh P, Smart HL, Gilmore I, Evans J, Hughes M, Garvey C, Sutton R and Neoptolemos JP: When is pancreatitis considered to be of biliary origin and what are the implications for management? Pancreatology 7: 131-141, 2007.

3. Youn YH, Lim HC, Jahng JH, Jang SI, You JH, Park JS, Lee SJ and Lee DK: The Increase in balloon size to over $15 \mathrm{~mm}$ does not affect the development of pancreatitis after endoscopic papillary large balloon dilatation for bile duct stone removal. Dig Dis Sci 56: 1572-1577, 2011.

4. Siqin D, Wang C, Zhou Z and Li Y: The key event of acute pancreatitis: Pancreatic duct obstruction and bile reflux, not a single one can be omitted. Med Hypothes 72: 589-591, 2009.

5. Neyrinck AM, Margagliotti S, Gomez C and Delzenne NM: Kupffer cell-derived prostaglandin E2 is involved in regulation of lipid synthesis in rat liver tissue. Cell Biochem Funct 22: 327-332, 2004.

6. Mäck C, Jungermann K, Götze O and Schieferdecker HL: Anaphylatoxin C5a actions in rat liver: Synergistic enhancement by C5a of lipopolysaccharide-dependent alpha(2)-macroglobulin gene expression in hepatocytes via IL-6 release from Kupffer cells. J Immunol 167: 3972-3979, 2001.

7. Wang JL, Chen Y, Song XQ, Lu ML, Zhao B, Ma L, Chen EZ and Mao EQ: Biliary tract external drainage protects against multiple organs injuries of severe acute pancreatitis rats via hemeoxygenas-1 upregulation. Pancreatology 17: 219-227, 2017.
8. Gonzalez RJ, Moore EE, Ciesla DJ, Biffl WL, Johnson JL and Silliman CC: Mesenteric lymph is responsible for post-hemorrhagic shock systemic neutrophil priming. J Trauma 51: 1069-1072, 2001.

9. Davidson MT, Deitch EA, Lu Q, Osband A, Feketeova E, Németh ZH, Haskó G and Xu DZ: A study of the biologic activity of trauma-hemorrhagic shock mesenteric lymph over time and the relative role of cytokines. Surgery 136: 32-41, 2004.

10. Fanous MY, Phillips AJ and Windsor JA: Mesenteric lymph: The bridge to future management of critical illness. JOP 8: 374-399, 2007.

11. Akbarshahi H, Sam A, Chen C, Rosendahl AH and Andersson R: Early activation of pulmonary TGF- $\beta 1 / \mathrm{Smad} 2$ signaling in mice with acute pancreatitis-associated acute lung injury. Mediators Inflamm 2014: 148029, 2014

12. Wang Z, Cheng Z, Cristofaro V, Li J, Xiao X, Gomez P, Ge R, Gong E, Strle K, Sullivan MP, et al: Inhibition of TNF-a improves the bladder dysfunction that is associated with type 2 diabetes. Diabetes 61: 2134-2145, 2012.

13. Fagone P, Mangano K, Di Marco R, Touil-Boukoffa C, Chikovan T, Signorelli S, Lombardo GA, Patti F, Mammana S and Nicoletti F: Expression of DNA methylation genes in secondary progressive multiple sclerosis. J Neuroimmunol 290: 66-69, 2016.

14. Li BZ, Guo B, Zhang HY, Liu J, Tao SS, Pan HF and Ye DQ: Therapeutic potential of HO-1 in autoimmune diseases. Inflammation 37: 1779-1788, 2014

15. Liu R and Yan X: Sulforaphane protects rabbit corneas against oxidative stress injury in keratoconus through activation of the Nrf-2/HO-1 antioxidant pathway. Int J Mol Med 42: 2315-2328, 2018.

16. Altavilla D, Famulari C, Passaniti M, Galeano M, Macrì A, Seminara P, Minutoli L, Marini H, Calò M, Venuti FS, et al: Attenuated cerulein-induced pancreatitis in nuclear factor-kappaB-deficient mice. Lab Invest 83: 1723-1732, 2003.

17. Ijaz T, Sun H, Pinchuk IV, Milewicz DM, Tilton RG and Brasier AR: Deletion of NF- $\kappa \mathrm{B} /$ RelA in angiotensin II-sensitive mesenchymal cells blocks aortic vascular inflammation and abdominal aortic aneurysm formation. Arterioscler Thromb Vasc Biol 37: 1881-1890, 2017.

18. Kusske AM, Rongione AJ, Ashley SW, McFadden DW and Reber HA: Interleukin-10 prevents death in lethal necrotizing pancreatitis in mice. Surgery 120: 284-289, 1996.

19. Gunjaca I, Zunic J, Gunjaca M and Kovac Z: Circulating cytokine levels in acute pancreatitis-model of SIRS/CARS can help in the clinical assessment of disease severity. Inflammation 35: 758-763, 2012.

20. Frossard JL, Steer ML and Pastor CM: Acute pancreatitis. Lancet 371: 143-152, 2008.

21. Petersen $\mathrm{OH}: \mathrm{Ca} 2+$ signaling in pancreatic acinar cells: Physiology and pathophysiology. Braz J Med Biol Res 42: 9-16, 2009.

22. Pandol SJ, Saluja AK, Imrie CW and Banks PA: Acute pancreatitis: Bench to the bedside. Gastroenterology 132: 1127-1151, 2007.

23. Leaphart CL and Tepas JJ III: The gut is a motor of organ system dysfunction. Surgery 141: 563-569, 2007.

24. Sharma D, Jakkampudi A, Reddy R, Reddy PB, Patil A, Murthy HVV, Rao GV, Reddy DN and Talukdar R: Association of systemic inflammatory and anti-inflammatory responses with adverse outcomes in acute pancreatitis: Preliminary results of an ongoing study. Dig Dis Sci 62: 3468-3478, 2017.

25. Petrov MS, Shanbhag S, Chakraborty M, Phillips AR and Windsor JA: Organ failure and infection of pancreatic necrosis as determinants of mortality in patients with acute pancreatitis. Gastroenterology 139: 813-820, 2010.

26. Pellegrini CA: Surgery for gallstone pancreatitis. Am J Surg 165: 515-518, 1993.

27. Jackson GD, Dai Y and Sewell WA: Bile mediates intestinal pathology in endotoxemia in rats. Infect Immun 68: 4714-4719, 2000.

28. Landahl P, Ansari D and Andersson R: Severe acute pancreatitis: Gut barrier failure, systemic inflammatory response, acute lung injury, and the role of the mesenteric lymph. Surg Infect (Larchmt) 16: 651-656, 2015.

29. Yang R, Miki K, Oksala N, Nakao A, Lindgren L, Killeen ME, Mennander A, Fink MP and Tenhunen J: Bile high-mobility group box 1 contributes to gut barrier dysfunction in experimental endotoxemia. Am J Physiol Regul Integr Comp Physiol 297: R362-R369, 2009.

30. Saluja SS, Kalayarasan R, Mishra PK, Srivastava S, Chandrasekar S and Godhi S: Chronic pancreatitis with benign biliary obstruction: Management issues. World J Surg 38: 2455-2459, 2014. 
31. Ramudo L, Manso MA and De Dios I: Biliary pancreatitis-associated ascitic fluid activates the production of tumor necrosis factor-alpha in acinar cells. Crit Care Med 33: 143-148, discussion 248, 2005.

32. Norman JG, Fink GW and Franz MG: Acute pancreatitis induces intrapancreatic tumor necrosis factor gene expression. Arch Surg 130: 966-970, 1995.

33. Yang ZY, Ling Y, Yin T, Tao J, Xiong JX, Wu HS and Wang CY: Delayed ethyl pyruvate therapy attenuates experimental severe acute pancreatitis via reduced serum high mobility group box 1 levels in rats. World J Gastroenterol 14: 4546-4550, 2008.

34. Musumeci D, Roviello GN and Montesarchio D: An overview on HMGB1 inhibitors as potential therapeutic agents in HMGB1-related pathologies. Pharmacol Ther 141: 347-357, 2014

35. Lee JC, Menacherry S, Diehl MC, Giffear MD, White CJ, Juba R, Bagarazzi ML, Muthumani K, Boyer J, Agarwal V, et al: Safety, bioavailability, and pharmacokinetics of VGX-1027-A novel oral anti-inflammatory drug in healthy human subjects. Clin Pharmacol Drug Dev 5: 91-101, 2016.

36. Aziz NM, Kamel MY and Rifaai RA: Effects of hemin, a heme oxygenase-1 inducer in L-arginine-induced acute pancreatitis and associated lung injury in adult male albino rats. Endocr Regul 51: 20-30, 2017
37. Song L, Li J, Yuan X, Liu W, Chen Z, Guo D, Yang F, Guo Q and Song H: Carbon monoxide-releasing molecule suppresses inflammatory and osteoclastogenic cytokines in nicotine- and lipopolysaccharide-stimulated human periodontal ligament cells via the heme oxygenase-1 pathway. Int J Mol Med 40: 1591-1601, 2017.

38. Mangano K, Cavalli E, Mammana S, Basile MS, Caltabiano R, Pesce A, Puleo S, Atanasov AG, Magro G, Nicoletti F and Fagone P: Involvement of the Nrf2/HO-1/CO axis and therapeutic intervention with the CO-releasing molecule CORM-A1, in a murine model of autoimmune hepatitis. J Cell Physiol 233: 4156-4165, 2018.

39. Tobiasch E, Günther L and Bach FH: Heme oxygenase-1 protects pancreatic beta cells from apoptosis caused by various stimuli. J Investig Med 49: 566-571, 2001.

40. Ribeiro MM, Klein D, Pileggi A, Molano RD, Fraker C, Ricordi C, Inverardi L and Pastori RL: Heme oxygenase-1 fused to a TAT peptide transduces and protects pancreatic beta-cells. Biochem Biophys Res Commun 305: 876-881, 2003.

41. Piao X, Liu B, Guo L, Meng F and Gao L: Picroside II shows protective function for severe acute pancreatitis in rats by preventing NF- $\kappa \mathrm{B}$-dependent autophagy. Oxid Med Cell Longev 2017: 7085709, 2017. 